\title{
CLASSIFICAÇÃO DAS ÁLGEBRAS DE LIE TRIDIMENSIONAIS
}

José Paulo Rodrigues da Silveira, Fernando Pereira de Sousa

Universidade Federal de Mato Grosso do Sul - UFMS, Campus de Três Lagoas. Bolsista do Grupo PET Conexões de Saberes - Matemática/CPTL/UFMS. E-mail: josepapt@hotmail.com

\section{RESUMO}

O trabalho apresenta resultados de um estudo sobre "Estruturas Algébricas com ênfase em elementos da Teoria De Lie" que foi desenvolvido como parte das atividades de pesquisa e apresentações de seminários, vinculado às disciplinas de Álgebra e Álgebra Linear, com o objetivo de um futuro aprofundamento na teoria de álgebras não comutativas. Durante o desenvolvimento do presente trabalho, foram estudados alguns conceitos de Álgebra Linear, relacionados com Espaços Vetoriais, além de alguns conceitos de álgebra, no que diz respeito a Grupos, Aneis, Corpos e Ideais. Em seguida, foram estudados as definições, proposições e teoremas necessários para a abordagem das Álgebras de Lie Solúveis e Nilpotentes, bem como a classificação de álgebras de Lie Tridimensionais.

Palavras - chave: Lie, Álgebras, Solúvel, Nilpotente, Tridimensional.

\section{CLASSIFICATION OF Lie ALGEBRA DIMENSIONAL}

\begin{abstract}
The paper presents results of a study on "Algebraic Structures with an emphasis on elements of Lie Theory " that was developed as part of research activities and seminar presentations, linked to the disciplines of Algebra and Linear Algebra, with a view to a future deepening in algebra theory does not commutative. During the development of this work, we studied some concepts of linear algebra, vector spaces related, and some algebra concepts, with regard to groups, rings, Bodies and ideals. Then, the settings were studied, and theorems propositions required for the approach of Lie algebras soluble and nilpotent as well as the classification of Lie algebras dimensional
\end{abstract}

Keywords: Lie, Álgebra, Soluble, Nilpotent, Three-dimensional. 
INTRODUÇÃO

Para uma melhor compreensão quanto á classificação das álgebras de Lie tridimensionais é necessário um conhecimento básico sobre:

Definição 1: Uma álgebra de Lie é um espaço vetorial a munido da operação colchete de Lie:

$$
\begin{aligned}
\mathfrak{a} \times \mathfrak{a} & \mapsto \mathfrak{a} \\
(\mathrm{X}, \mathrm{Y}) & \mapsto[\mathrm{X}, \mathrm{Y}]
\end{aligned}
$$

O colchete de Lie satisfaz às condições:

1. O colchete de Lie é bilinear, isto é,

$$
\begin{aligned}
& {[\mathrm{aX}+\mathrm{bY}, \mathrm{Z}]=\mathrm{a}[\mathrm{X}, \mathrm{Z}]+\mathrm{b}[\mathrm{Y}, \mathrm{Z}]} \\
& {[\mathrm{Z}, \mathrm{aX}+\mathrm{bY}]=\mathrm{a}[\mathrm{Z}, \mathrm{X}]+\mathrm{b}[\mathrm{Z}, \mathrm{Y}]}
\end{aligned}, \quad \forall \mathrm{a}, \mathrm{b} \in \mathbb{R} \text { e } \forall \mathrm{X}, \mathrm{Y}, \mathrm{Z} \in \mathfrak{a} .
$$

2. O colchete de Lie é antissimétrico, isto é, $[X, X]=0, \forall X \in \mathfrak{a}$.

3. A identidade de Jacobi é satisfeita $\forall X, Y, Z \in \mathfrak{a}$ :

$$
[\mathrm{X},[\mathrm{Y}, \mathrm{Z}]]+[\mathrm{Y},[\mathrm{Z}, \mathrm{X}]]+[\mathrm{Z},[\mathrm{X}, \mathrm{Y}]]=0
$$

Definição 2: Seja B um subconjunto de $\mathfrak{a}$, chamamos de centralizador de $B$ em $\mathfrak{a}$, ao conjunto $\mathrm{z}(\mathrm{B})=\{\mathrm{X} \in \mathfrak{a} /[\mathrm{X}, \mathrm{Y}]=0, \forall \mathrm{Y} \in \mathrm{B}\}$.

Definição 3: Se a é uma álgebra de Lie, então o centro de a será denotado por:

$$
c(\mathfrak{a})=\{X \in \mathfrak{a} /[X, Y]=0, \forall Y \in \mathfrak{a}\} .
$$

Definição 4: Seja a uma álgebra de Lie. Dizemos que $\mathfrak{a}^{\prime}$ é uma álgebra derivada de a se: $\mathfrak{a}^{\prime}=<\{[X, Y] / X, Y \in \mathfrak{a}\}>$.

\section{ÁLGEBRAS DE LIE TRIDIMENSIONAIS}

Seja a uma álgebra de Lie tridimensional e seja $\mathfrak{a}^{\prime}$ sua álgebra derivada. Classificaremos esta álgebra através das dimensões da álgebra derivada $\mathfrak{a}^{\prime}$.

Teorema. Seja a uma álgebra de Lie tridimensional e tal que sua álgebra derivada $\mathfrak{a}^{\prime}$ é nula. Então, a é abeliana. 
Demonstração: Suponhamos que $\operatorname{dim}\left(\mathfrak{a}^{\prime}\right)=0$, isto é, $\mathfrak{a}^{\prime}=\{0\}$. Neste caso, tem-se que a é uma álgebra abeliana.

Agora, analisaremos o caso em que $\operatorname{dim}\left(\mathfrak{a}^{\prime}\right)=1$. Este caso se dividirá em duas etapas, a primeira considerando $\mathfrak{a}^{\prime} \subset c(\mathfrak{a})$ e outra considerando que $\mathfrak{a}^{\prime}$ não está contido em $c(\mathfrak{a})$. Para $\mathfrak{a}^{\prime} \subset c(\mathfrak{a})$ temos o seguinte resultado sobre classificação de álgebras de Lie tridimensionais:

Teorema. Seja a uma álgebra de Lie tridimensional cuja álgebra derivada $\mathfrak{a}^{\prime}$ é unidimensional. Suponha que $\mathfrak{a}^{\prime} \subset c(\mathfrak{a})$. Então, existe uma base $\{\mathrm{X}, \mathrm{Y}, \mathrm{Z}\}$ de $\mathfrak{a}$ tal que $[Y, Z]=X,[X, Y]=0$ $\mathrm{e}[\mathrm{X}, \mathrm{Z}]=0$.

Demonstração: Sejam $\{X\}$ e $\left\{X, Y_{1}, Z\right\}$ bases de $\mathfrak{a}^{\prime}$ e $\mathfrak{a}$, respectivamente. Como $\mathfrak{a}^{\prime} \subset c(\mathfrak{a})$, temos que para qualquer $U \in \mathfrak{a}^{\prime}$ tem-se que $[\mathrm{U}, \mathrm{W}]=0, \forall W \in \mathfrak{a}$. Como $\{X\}$ é base de $\mathfrak{a}^{\prime}$ temos $[\mathrm{X}, \mathrm{W}]=0, \forall W \in \mathfrak{a}, \mathrm{e}$, em $\left[X, Y_{1}\right]=0$ e $[X, Z]=0$, pois $Y_{1}, Z \in \mathfrak{a}$. Já que $\left[Y_{1}, Z\right] \in \mathfrak{a}^{\prime}$, segue que $\left[Y_{1}, Z\right]=a X, a \neq 0$. Verifiquemos que $a$ é realmente não nulo. Caso contrário, $\left[Y_{1}, Z\right]=0$. Como $\left[X, Y_{1}\right]=0=[X, Z]$ temos que $\forall U, V \in \mathfrak{a}$, vale

$$
[U, V]=\left[a X+b Y_{1}+c Z, \alpha X+\beta Y_{1}+\gamma Z\right]=0 .
$$

Concluímos então que $\operatorname{dim}\left(\mathfrak{a}^{\prime}\right)=0$, contradizendo a hipótese. Definamos $\mathrm{Y}=\frac{1}{a} Y_{1}$. Então, $\{X, Y, Z\}$ também é uma base de a e

$$
[X, Y]=0=[X, Z] \text { e }[Y, Z]=\frac{1}{a}\left[Y_{1}, Z\right]=\frac{1}{a} a X=X .
$$

Agora, para analisar o caso em que $\mathfrak{a}^{\prime}$ não está contido em $c(\mathfrak{a})$ precisamos dos dois lemas a seguir.

Lema: Seja $\mathfrak{a}$ uma álgebra de Lie tridimensional tal que $\mathfrak{a}^{\prime}$ é unidimensional. Suponha que $\mathfrak{a}$ possua uma subálgebra bidimensional $\mathfrak{b}$ que não é abeliana. Então, $\mathfrak{b}$ é um ideal de $\mathfrak{a}$.

Lema. Seja a uma álgebra de Lie tridimensional tal que $\mathfrak{a}^{\prime}$ é unidimensional. Se $\mathfrak{a}^{\prime}$ não está contido em $c(\mathfrak{a})$, então existe uma subálgebra bidimensional de a que não é abeliana. 
Veremos a seguir, uma classificação das álgebras de Lie de dimensão três, cuja álgebra derivada $\mathfrak{a}^{\prime}$ é unidimensional e não está contida em $c(\mathfrak{a})$.

Teorema. Seja a uma álgebra de Lie tridimensional tal que $\mathfrak{a}^{\prime}$ é unidimensional. Se $\mathfrak{a}^{\prime}$ não está contido em $c(\mathfrak{a})$, então existe uma base $\{X, Y, Z\}$ de a tal que $[X, Y]=X,[X, Z]=0$ e $[Y, Z]=0$.

Demonstração: Pelo lema anterior, temos que a possui uma subálgebra bidimensional não abeliana $\mathfrak{b}$. Seja $\{X, Y\}$ a base canônica de $\mathfrak{b} \operatorname{com}[X, Y]=X$. Pelos lemas anteriores, temos que $\mathfrak{b}$ é um ideal de $\mathfrak{a}$. Logo, $\mathfrak{a}=\mathfrak{b} \oplus \mathrm{z}(\mathfrak{b})$, onde $z(\mathfrak{b})$ é o centralizador de $\mathfrak{b}$ em $\mathfrak{a}$. Completemos a base $\{X, Y\}$ de $\mathfrak{b}$ para obtermos a base $\{X, Y, Z\}$ de $\mathfrak{a}$. Como $\mathfrak{a}=\mathfrak{b} \oplus \mathbf{z}(\mathfrak{b})$ e $\{X, Y\}$ gera $\mathfrak{b}$, temos que $\mathrm{Z} \in \mathrm{z}(\mathfrak{b})$. Decorre daí que $[Z, X]=0$ e $[Z, Y]=0$.

À seguir, apresentaremos o caso em que a álgebra derivada $\mathfrak{a}^{\prime}$ é bidimensional:

Lema: Seja a uma álgebra de Lie tridimensional tal que $\mathfrak{a}^{\prime}$ é bidimensional. Suponha que $\mathfrak{a}$ possui uma subálgebra não abeliana bidimensional $\mathfrak{b}$. Então, o ideal $\mathfrak{a}^{\prime}$ é diferente de $\mathfrak{b}$.

Demonstração: Suponhamos por absurdo que $\mathfrak{a}^{\prime}=\mathfrak{b}$. Tomemos uma base $\{X, Y\}$ de $\mathfrak{b}$ com $[X, Y]=Y$. Como $\mathfrak{b}$ é ideal, temos que $\mathfrak{a}=\mathfrak{b} \oplus \mathrm{z}(\mathfrak{b})$ e, daí,

$$
\begin{gathered}
\mathfrak{a}^{\prime}=[\mathfrak{b} \oplus \mathrm{z}(\mathfrak{b}), \mathfrak{b} \oplus \mathrm{z}(\mathfrak{b})] \\
\therefore \mathfrak{a}^{\prime}=[\mathfrak{b}, \mathfrak{b}]+[\mathfrak{b}, \mathrm{z}(\mathfrak{b})]+[z(\mathfrak{b}), \mathfrak{b}]+[z(\mathfrak{b}), z(\mathfrak{b})] \\
\therefore \mathfrak{a}^{\prime}=[\mathfrak{b}, \mathfrak{b}]+[z(\mathfrak{b}), z(\mathfrak{b})] .
\end{gathered}
$$

Como $\operatorname{dim}(\mathfrak{b})=2$ e $\mathfrak{a}=\mathfrak{b} \oplus \mathrm{z}(\mathfrak{b})$, temos que $\operatorname{dim}(\mathrm{z}(\mathfrak{b}))=1$. Daí, concluímos que $\mathrm{z}(\mathfrak{b})$ é abeliano e, portanto, $[z(\mathfrak{b}), z(\mathfrak{b})]=0$. Assim, da igualdade $\mathfrak{a}^{\prime}=[\mathfrak{b}, \mathfrak{b}]+[z(\mathfrak{b}), z(\mathfrak{b})]$, temos que $\mathfrak{a}^{\prime}=\mathfrak{b}^{\prime}$. Como $\mathfrak{b}^{\prime}=[\mathfrak{b}, \mathfrak{b}]$ é gerado por $Y$, temos que $\mathfrak{a}^{\prime}$ é unidimensional, o que contradiz a hipótese.

Definição: A aplicação ad abaixo é chamada de representação adjunta da álgebra de Lie $\mathfrak{a}$

$$
\begin{gathered}
\operatorname{ad}(X): \mathfrak{a} \rightarrow \mathfrak{a} \\
X \mapsto \operatorname{ad}(\mathrm{X})
\end{gathered}
$$

Lema. Seja a uma álgebra de Lie tridimensional tal que sua álgebra derivada $\mathfrak{a}^{\prime}$ é bidimensional. Então, $\operatorname{ad}(Z): \mathfrak{a}^{\prime} \rightarrow \mathfrak{a}^{\prime}$ é um isomorfismo $\forall Z \in \mathfrak{a}, Z \neq \mathfrak{a}^{\prime}$. 
O resultado a seguir classifica as álgebras de Lie Tridimensionais, cuja álgebra derivada é bidimensional.

Teorema. Seja a uma álgebra de Lie tridimensional tal que sua álgebra derivada $\mathfrak{a}^{\prime}$ é bidimensional. Então, existe uma base $\{\mathrm{X}, \mathrm{Y}, \mathrm{Z}\}$ de $\mathfrak{a}$ e escalares $\alpha, \beta, \gamma, \delta$ tais que $[X, Y]=$ $0,[Z, X]=\alpha X+\beta Y,[Z, Y]=\gamma X+\delta Y$, e $W=\left(\begin{array}{ll}\alpha & \beta \\ \gamma & \delta\end{array}\right)$ é uma matriz invertível.

Demonstração: Por intermédio do lema anterior, como $\mathfrak{a}^{\prime}$ é abeliana, tomemos uma base $\{\mathrm{X}, \mathrm{Y}\}$ de $\mathfrak{a}^{\prime} \operatorname{com}[\mathrm{X}, \mathrm{Y}]=0$ e estendamos esta base a uma base $\{\mathrm{X}, \mathrm{Y}, \mathrm{Z}\}$ de $\mathfrak{a}$. Ainda pelo lema anterior, $\{[\mathrm{X}, \mathrm{Z}],[\mathrm{Y}, \mathrm{Z}]\}$ também é uma base de $\mathfrak{a}^{\prime}$, assim $[X, Z]=\alpha X+\beta Y$ e, do mesmo modo, $[\mathrm{Y}, \mathrm{Z}]=\gamma X+\delta Y$, uma vez que $\{X, Y\}$ também é base de $\mathfrak{a}^{\prime}$. Como ad(Z) é um isomorfismo, temos que $\mathrm{U}=\left(\begin{array}{ll}\alpha & \gamma \\ \beta & \delta\end{array}\right)$ é invertível, pois é a matriz do isomorfismo ad(Z).

Assim, como a transposta de uma matriz invertível também é invertível,

$$
U^{T}=W=\left(\begin{array}{ll}
\alpha & \beta \\
\gamma & \delta
\end{array}\right) \text { é invertível. }
$$

Definição. Duas matrizes $A$ e $B$ são chamadas cogradientes se existe uma matriz invertível $\mathrm{N}$ e um número real $\mathrm{p} \neq 0$ tal que $\mathrm{B}=\mathrm{pN}^{\mathrm{T}} \mathrm{AN}$.

Usaremos a notação $A \sim B$ para denotar que $A$ é cogradientes a $B$.

Proposição. A relação $A$ é cogradientes a $B(A \sim B)$ é uma relação de equivalência.

A proposição a seguir nos mostra que no conjunto das matrizes simétricas e invertíveis só existem duas classes de equivalência de matrizes cogradientes.

Proposição. Se $A$ é uma matriz $3 \times 3$ real, simétrica e invertível, então $A$ é cogradientes a

$$
\left(\begin{array}{lll}
1 & 0 & 0 \\
0 & 1 & 0 \\
0 & 0 & 1
\end{array}\right) \text { ou a }\left(\begin{array}{ccc}
-1 & 0 & 0 \\
0 & 1 & 0 \\
0 & 0 & 1
\end{array}\right) \text {. }
$$

Demonstração: Seja A uma matriz real, simétrica e invertível. Pelo teorema espectral, temos que existe uma matriz ortogonal $\mathrm{N}$ tal que $\mathrm{N}^{T} \mathrm{NA}$ é uma matriz diagonal, ou seja, 


$$
\mathrm{N}^{T} \mathrm{AN}=\left(\begin{array}{ccc}
\alpha & 0 & 0 \\
0 & \beta & 0 \\
0 & 0 & \gamma
\end{array}\right), \operatorname{com} \alpha, \beta, \gamma \in \mathbb{R}
$$

Como $A$ é invertível e $\operatorname{det}(A) \neq 0$, temos que $\operatorname{det}\left(\mathrm{N}^{T} \mathrm{AN}\right)=\alpha \beta \gamma \neq 0$. Multiplicando $\mathrm{N}^{T} \mathrm{NA}$ por $\gamma^{-1}$, obtemos: $\left(\begin{array}{ccc}\frac{\alpha}{\gamma} & 0 & 0 \\ 0 & \frac{\beta}{\gamma} & 0 \\ 0 & 0 & 1\end{array}\right)$. Tomando $\frac{\alpha}{\gamma}=\alpha^{\prime}, \mathrm{e} \frac{\beta}{\gamma}=\beta^{\prime}$, temos que $A$ é cogradientes a

$$
\mathrm{B}=\left(\begin{array}{ccc}
\alpha^{\prime} & 0 & 0 \\
0 & \beta^{\prime} & 0 \\
0 & 0 & 1
\end{array}\right)
$$

Mostraremos que a matriz $B$ é cogradiente a:

$$
C=\left(\begin{array}{lll}
1 & 0 & 0 \\
0 & 1 & 0 \\
0 & 0 & 1
\end{array}\right) \text { ou a } D=\left(\begin{array}{ccc}
-1 & 0 & 0 \\
0 & 1 & 0 \\
0 & 0 & 1
\end{array}\right)
$$

De fato, para mostrar isto, seja $N=\left(\begin{array}{ccc}x & 0 & 0 \\ 0 & y & 0 \\ 0 & 0 & z\end{array}\right)$.

Para simplificarmos a notação, troquemos $\alpha^{\prime}$ por $\alpha$ e $\beta^{\prime}$ por $\beta$. Dessa forma, $\mathrm{N}^{T} \mathrm{BN}=$

$$
\left(\begin{array}{ccc}
x^{2} \alpha & 0 & 0 \\
0 & y^{2} \beta & 0 \\
0 & 0 & z^{2}
\end{array}\right)
$$

Para concluir a demonstração, consideraremos todas as possibilidades para os sinais de $\alpha$ e $\beta$.

- Se $\alpha>0$ e $\beta>0$, tomamos a matriz $N$ tal que $x=\frac{1}{\sqrt{\alpha}}, y=\frac{1}{\sqrt{\beta}}$ e $z=1$. Daí,

$$
\mathrm{N}^{T} \mathrm{BN}=\left(\begin{array}{lll}
1 & 0 & 0 \\
0 & 1 & 0 \\
0 & 0 & 1
\end{array}\right)=C, \text { ou seja, } \mathrm{B} \sim \mathrm{C} .
$$

- Se $\alpha<0$ e $\beta>0$, tomamos a matriz $N$ tal que $x=\frac{1}{\sqrt{-\alpha}}, y=\frac{1}{\sqrt{\beta}}$ e $z=1$. Daí,

$$
\mathrm{N}^{T} \mathrm{BN}=\left(\begin{array}{ccc}
-1 & 0 & 0 \\
0 & 1 & 0 \\
0 & 0 & 1
\end{array}\right)=D, \text { ou seja, } \mathrm{B} \sim \mathrm{D}
$$

- Se $\alpha>0$ e $\beta<0$, tomamos a matriz $N$ tal que $x=\frac{1}{\sqrt{\alpha}}, y=\frac{1}{\sqrt{-\beta}}$ e $z=1$. Daí,

$$
\mathrm{N}^{T} \mathrm{BN}=\left(\begin{array}{ccc}
1 & 0 & 0 \\
0 & -1 & 0 \\
0 & 0 & 1
\end{array}\right) \text {, ou seja, B é cogradientes a } \mathrm{E}=\left(\begin{array}{ccc}
1 & 0 & 0 \\
0 & -1 & 0 \\
0 & 0 & 1
\end{array}\right)
$$

Agora, observe que se tomarmos $N=\left(\begin{array}{lll}0 & 1 & 0 \\ 1 & 0 & 0 \\ 0 & 0 & 1\end{array}\right)$, teremos que 


$$
(-1)\left(\begin{array}{lll}
0 & 1 & 0 \\
1 & 0 & 0 \\
0 & 0 & 1
\end{array}\right)\left(\begin{array}{ccc}
1 & 0 & 0 \\
0 & -1 & 0 \\
0 & 0 & 1
\end{array}\right)\left(\begin{array}{lll}
0 & 1 & 0 \\
1 & 0 & 0 \\
0 & 0 & 1
\end{array}\right)=\left(\begin{array}{ccc}
-1 & 0 & 0 \\
0 & 1 & 0 \\
0 & 0 & 1
\end{array}\right) .
$$

Dessa forma, E DD.

- Se $\alpha<0$ e $\beta<0$, tomamos a matriz $N$ tal que $x=\frac{1}{\sqrt{-\alpha}}, y=\frac{1}{\sqrt{-\beta}}$ e $z=1$. Daí,

$$
\begin{aligned}
& \mathrm{N}^{T} \mathrm{BN}=\left(\begin{array}{ccc}
-1 & 0 & 0 \\
0 & -1 & 0 \\
0 & 0 & -1
\end{array}\right) \text {, ou seja, } \mathrm{B} \sim \mathrm{F}=\left(\begin{array}{ccc}
-1 & 0 & 0 \\
0 & -1 & 0 \\
0 & 0 & -1
\end{array}\right) \text {. Agora, tomemos } N= \\
& \left(\begin{array}{lll}
0 & 0 & 1 \\
0 & 1 & 0 \\
1 & 0 & 0
\end{array}\right)
\end{aligned}
$$

Notemos que:

$$
(-1)\left(\begin{array}{lll}
0 & 0 & 1 \\
0 & 1 & 0 \\
1 & 0 & 0
\end{array}\right)\left(\begin{array}{ccc}
-1 & 0 & 0 \\
0 & -1 & 0 \\
0 & 0 & -1
\end{array}\right)\left(\begin{array}{lll}
0 & 0 & 1 \\
0 & 1 & 0 \\
1 & 0 & 0
\end{array}\right)=\left(\begin{array}{ccc}
-1 & 0 & 0 \\
0 & 1 & 0 \\
0 & 0 & 1
\end{array}\right) .
$$

Daí, temos que F D.

Com o teorema a seguir, encerraremos as classificações das álgebras de Lie tridimensionais:

Teorema: Seja a uma álgebra de Lie tridimensional tal que sua álgebra derivada $\mathfrak{a}^{\prime}$ também é tridimensional, ou seja, $\mathfrak{a}=\mathfrak{a}^{\prime}$. Então, existem exatamente duas classes de álgebras de Lie tridimensionais distintas. Uma com colchetes entre os elementos da base dados por $[\mathrm{Y}, \mathrm{Z}]=$ $\mathrm{X} ;[\mathrm{Z}, \mathrm{X}]=\mathrm{Y}$ e $[\mathrm{X}, \mathrm{Y}]=\mathrm{Z}$ e a outra com colchetes dados por $[\mathrm{Y}, \mathrm{Z}]=-\mathrm{X} ;[\mathrm{Z}, \mathrm{X}]=\mathrm{Y}$ e $[\mathrm{X}, \mathrm{Y}]=\mathrm{Z}$.

Demonstração: Seja $\left\{\mathrm{X}_{1}, X_{2}, X_{3}\right\}$ uma base de a. Não é difícil demonstrar que $\left[X_{2}, X_{3}\right]=Y_{1}$; $\left[X_{3}, X_{1}\right]=Y_{2}$ e $\left[X_{1}, X_{2}\right]=Y_{3}$ geram $\mathfrak{a}^{\prime}$ e, portanto, constituem uma base de $\mathfrak{a}^{\prime}$. Como $\mathfrak{a}=\mathfrak{a}^{\prime}$, temos que $\left\{Y_{1}, Y_{2}, Y_{3}\right\}$ também é uma base de $a$. Denotaremos por

$$
A=\left(\begin{array}{lll}
\alpha_{11} & \alpha_{21} & \alpha_{31} \\
\alpha_{12} & \alpha_{22} & \alpha_{32} \\
\alpha_{13} & \alpha_{23} & \alpha_{33}
\end{array}\right)
$$

A matriz mudança de base de $\left\{\mathrm{X}_{1}, X_{2}, X_{3}\right\}$ para a base $\left\{Y_{1}, Y_{2}, Y_{3}\right\}$. Sabemos que $A$ é invertível e mostremos, através da identidade de Jacobi, que a matriz A é simétrica. Com efeito, pela identidade de Jacobi, temos que

$$
\begin{array}{r}
{\left[\mathrm{X}_{1},\left[X_{2}, X_{3}\right]\right]+\left[X_{3}\left[X_{1}, X_{2}\right]\right]+\left[X_{2},\left[X_{3}, X_{1}\right]\right]=0, \text { mas }} \\
{\left[\mathrm{X}_{1}\left[X_{2}, X_{3}\right]\right]+\left[X_{3}\left[X_{1}, X_{2}\right]\right]+\left[X_{2},\left[X_{3}, X_{1}\right]\right]=\left[X_{1}, Y_{1}\right]+\left[X_{3}, Y_{3}\right]+\left[X_{2}, Y_{2}\right]}
\end{array}
$$


$=\left[X_{1}, \alpha_{11} X_{1}+\alpha_{12} X_{2}+\alpha_{13} X_{3}\right]+\left[X_{3}, \alpha_{31} X_{1}+\alpha_{32} X_{2}+\alpha_{33} X_{3}\right]+\left[X_{2}, \alpha_{21} X_{1}+\alpha_{21} X_{2}+\alpha_{23} X_{3}\right]$

$=\alpha_{12}\left[X_{1}, X_{2}\right]+\alpha_{13}\left[X_{1}, X_{3}\right]+\alpha_{31}\left[X_{3}, X_{1}\right]+\alpha_{32}\left[X_{3}, X_{2}\right]+\alpha_{21}\left[X_{2}, X_{1}\right]+\alpha_{23}\left[X_{2}, X_{3}\right]$

$=\left(\alpha_{12}-\alpha_{21}\right)\left[X_{1}, X_{2}\right]+\left(\alpha_{31}-\alpha_{13}\right)\left[X_{3}, X_{1}\right]+\left(\alpha_{23}-\alpha_{32}\right)\left[X_{2}, X_{3}\right]$

Como $\left\{Y_{1}, Y_{2}, Y_{3}\right\}$ é linearmente independente, a identidade de Jacobi nos diz que $\alpha_{12}-\alpha_{21}=\alpha_{31}-\alpha_{13}=\alpha_{23}-\alpha_{32}=0$, e assim, $\alpha_{12}=\alpha_{21}, \alpha_{31}=\alpha_{13}$ e $\alpha_{23}=\alpha_{32}$, mostrando que a matriz $A$ é simétrica.

Consideremos agora uma outra base de a que denotaremos por $\left\{\overline{\mathrm{X}_{1}}, \overline{X_{2}}, \overline{X_{3}}\right\}$. Temos que

$$
\begin{aligned}
& \overline{\mathrm{X}_{1}}=\beta_{11} X_{1}+\beta_{12} X_{2}+\beta_{13} X_{3} \\
& \overline{\mathrm{X}_{2}}=\beta_{21} X_{1}+\beta_{22} X_{2}+\beta_{23} X_{3} \\
& \overline{\mathrm{X}_{3}}=\beta_{31} X_{1}+\beta_{32} X_{2}+\beta_{33} X_{3}
\end{aligned}
$$

e a matriz

$$
B=\left(\begin{array}{lll}
\beta_{11} & \beta_{12} & \beta_{13} \\
\beta_{21} & \beta_{22} & \beta_{23} \\
\beta_{31} & \beta_{32} & \beta_{33}
\end{array}\right) \text { é invertível. }
$$

Definamos $\overline{\mathrm{Y}_{1}}=\left[\overline{\mathrm{X}_{2}}, \overline{\mathrm{X}_{3}}\right], \overline{\mathrm{Y}_{2}}=\left[\overline{\mathrm{X}_{3}}, \overline{\mathrm{X}_{1}}\right], \overline{\mathrm{Y}_{3}}=\left[\overline{\mathrm{X}_{1}}, \overline{\mathrm{X}_{2}}\right]$.

Para qualquer permutação cíclica $(\mathrm{i}, \mathrm{j}, \mathrm{k})$ de $(1,2,3)$ temos que

$$
\begin{aligned}
\overline{\mathrm{Y}}_{l} & =\left[\overline{\mathrm{X}}, \overline{\mathrm{X}_{k}}\right]=\left[\beta_{j 1} X_{1}+\beta_{j 2} X_{2}+\beta_{j 3} X_{3}, \beta_{k 1} X_{1}+\beta_{k 2} X_{2}+\beta_{k 3} X_{3}\right] \\
& =\left(\beta_{j 2} \beta_{k 3}-\beta_{j 3} \beta_{k 2}\right) \mathrm{Y}_{1}+\left(\beta_{j 3} \beta_{k 1}-\beta_{j 1} \beta_{k 3}\right) \mathrm{Y}_{2}+\left(\beta_{j 1} \beta_{k 2}-\beta_{j 2} \beta_{k 1}\right) \mathrm{Y}_{3} \\
& =\gamma_{i 1} \mathrm{Y}_{1}+\gamma_{i 2} \mathrm{Y}_{2}+\gamma_{i 3} \mathrm{Y}_{3} .
\end{aligned}
$$

Assim,

$$
\left(\begin{array}{lll}
\gamma_{11} & \gamma_{12} & \gamma_{13} \\
\gamma_{21} & \gamma_{22} & \gamma_{23} \\
\gamma_{31} & \gamma_{32} & \gamma_{33}
\end{array}\right)=\left(B^{T}\right)^{-1} \operatorname{det}\left(B^{T}\right) \text { que é a matriz adjunta de } B^{T} .
$$

A matriz mudança de base de $\left\{\mathrm{X}_{1}, X_{2}, X_{3}\right\}$ para a base $\left\{Y_{1}, Y_{2}, Y_{3}\right\}$ é $A$ e a matriz mudança de base de $\left\{\overline{\mathrm{X}_{1}}, \overline{X_{2}}, \overline{X_{3}}\right\}$ para $\left\{\mathrm{X}_{1}, X_{2}, X_{3}\right\}$ é $\left(B^{T}\right)^{-1}$. Portanto, se $\overline{\mathrm{A}}$ é a matriz $\left(\overline{\alpha_{\imath \jmath}}\right)$ tal que $\overline{\mathrm{Y}}_{\iota}=$ $\bar{\alpha}_{i 1} \overline{\mathrm{X}}_{1}+\bar{\alpha}_{i 2} \overline{\mathrm{X}}_{2}+\bar{\alpha}_{i 3} \overline{\mathrm{X}}_{3}$ tem-se que

$$
\overline{\mathrm{A}}=\operatorname{det}\left(B^{T}\right)\left(B^{T}\right)^{-1} A B^{-1}
$$

Logo $A$ e $\overline{\mathrm{A}}$ são matrizes simétricas e cogradientes. Daí, $A$ (ou $\overline{\mathrm{A}}$ ) é cogradientes a $C$ ou a $D$ onde

$$
C=\left(\begin{array}{lll}
1 & 0 & 0 \\
0 & 1 & 0 \\
0 & 0 & 1
\end{array}\right) \text { e } D=\left(\begin{array}{ccc}
-1 & 0 & 0 \\
0 & 1 & 0 \\
0 & 0 & 1
\end{array}\right)
$$


Observemos que no caso da matriz $C$ obtemos a primeira classe de álgebras do enunciado. Analogamente, para a matriz $D$ obtemos a segunda classe de álgebras.

Exemplo. Um exemplo de álgebra de Lie tridimensional cuja álgebra derivada $\mathfrak{a}^{\prime}=\mathfrak{a}$ é a álgebra $\operatorname{sl}(2, \mathbb{R})=\{A \in M(n \times n, \mathbb{R}) / \operatorname{tr}(A)=0\}$.

De fato, os elementos de $\operatorname{sl}(2, \mathbb{R})$ são da forma $\left(\begin{array}{cc}a & b \\ c & -a\end{array}\right)$. Vamos analisar agora a sua álgebra derivada.

$$
\begin{gathered}
\operatorname{sl}(2, \mathbb{R})^{\prime}=\{[X, Y] / X, Y \in \operatorname{sl}(2, \mathbb{R})\} \\
\text { Seja } X=\left(\begin{array}{cc}
a_{1} & b_{1} \\
c_{1} & -a_{1}
\end{array}\right) \text { e } Y=\left(\begin{array}{cc}
a_{2} & b_{2} \\
c_{2} & -a_{2}
\end{array}\right) \text {, então } \\
{[X, Y]=\left(\begin{array}{cc}
a_{1} & b_{1} \\
c_{1} & -a_{1}
\end{array}\right)\left(\begin{array}{cc}
a_{2} & b_{2} \\
c_{2} & -a_{2}
\end{array}\right)-\left(\begin{array}{cc}
a_{2} & b_{2} \\
c_{2} & -a_{2}
\end{array}\right)\left(\begin{array}{cc}
a_{1} & b_{1} \\
c_{1} & -a_{1}
\end{array}\right)} \\
=\left(\begin{array}{cc}
b_{1} c_{2}-b_{2} c_{1} & -2 a_{2} b_{1} \\
2 c_{1} a_{2}-2 a_{1} c_{2} & -b_{1} c_{2}+b_{2} c_{1}
\end{array}\right) .
\end{gathered}
$$

Assim, concluímos que $\operatorname{sl}(2, \mathbb{R})^{\prime}$ possui dimensão 3 , pois a primeira e a quarta entrada da matriz acima são múltiplos. Portanto, $\operatorname{sl}(2, \mathbb{R})=\operatorname{sl}(2, \mathbb{R})^{\prime}$.

O objetivo deste estudo é o aprofundamento na teoria de álgebras não comutativas.

\section{METODOLOGIA}

O trabalho é resultado de uma pesquisa teórica desenvolvida nos anos de 2014 e 2015, embasada no livro "Estruturas Algébricas com ênfase em elementos da Teoria de Lie", desenvolvido através de discussões do tema com o orientador e apresentações de seminários como parte das atividades do programa PET Conexões de Saberes Matemática - UFMS/CPTL no estudo da Teoria De Lie. O trabalho incluiu uma etapa de leitura e resoluções de exercícios, desenvolvimento das atividades propostas e a tabulação dos resultados obtidos. O estudo e as atividades desenvolvidas foram avaliados através da apresentação de seminários de discussão.

\section{RESULTADOS}

Foram obtidos resultados importantes para a classificação de Álgebras de Lie Tridimensionais, o que permitiu fazer aprofundamentos no tema que serão temas a serem apresentados no TCC do curso de graduação do aluno e em apresentações de trabalhos de pesquisas futuras. 
CONCLUSÃO

Através do trabalho foram obtidos resultados que permitem um aprofundamento em estudos sobre a 'Teoria de Lie', bem como uma iniciação em conteúdos aprofundados da Álgebra não comutativa, possibilitando assim um maior conhecimento sobre áreas diversas de matemática.

\section{BIBLIOGRAFIA}

BARROS, C.J.B; SANTANA A.J. Estruturas Algébricas Com Ênfase em Elementos da Teoria de Lie. Maringá: Eduem, 2011.

COELHO, F. U.; LOURENÇO, M. L. Um Curso de Álgebra Linear. 2ª ed. São Paulo: Edusp, 2010.

HEFEZ A.; VILLELA, M. L. T. Introdução à Álgebra Linear. 1a ed. Rio de Janeiro: SBM, 2012. 